\title{
A!
}

This is an electronic reprint of the original article.

This reprint may differ from the original in pagination and typographic detail.

Lehtinen, Jyri J.; Spada, Federico; Käpylä, Maarit J.; Olspert, Nigul; Käpylä, Petri J.

\section{Common dynamo scaling in slowly rotating young and evolved stars}

Published in:

Nature Astronomy

DOI:

$10.1038 / s 41550-020-1039-x$

Published: 01/07/2020

Document Version

Publisher's PDF, also known as Version of record

Please cite the original version:

Lehtinen, J. J., Spada, F., Käpylä, M. J., Olspert, N., \& Käpylä, P. J. (2020). Common dynamo scaling in slowly rotating young and evolved stars. Nature Astronomy, 4(7), 658-662. https://doi.org/10.1038/s41550-020-1039-x

This material is protected by copyright and other intellectual property rights, and duplication or sale of all or part of any of the repository collections is not permitted, except that material may be duplicated by you for your research use or educational purposes in electronic or print form. You must obtain permission for any other use. Electronic or print copies may not be offered, whether for sale or otherwise to anyone who is not an authorised user. 


\title{
Common dynamo scaling in slowly rotating young and evolved stars
}

\author{
Jyri J. Lehtinen $\circledast^{1,2} \llbracket$, Federico Spada', Maarit J. Käpylä ${ }^{1,2}$, Nigul Olspert ${ }^{1}{ }^{1}$ and Petri J. Käpylä $\oplus^{2,3}$
}

\begin{abstract}
One interpretation of the activity and magnetism of late-type stars is that these both intensify with decreasing Rossby number up to a saturation level ${ }^{1-3}$, suggesting that stellar dynamos depend on both rotation and convective turbulence ${ }^{4}$. Some studies have claimed, however, that rotation alone suffices to parametrize this scaling adequately ${ }^{5,6}$. Here, we tackle the question of the relevance of turbulence to stellar dynamos by including evolved, post-main-sequence stars in the analysis of the rotation-activity relation. These stars rotate very slowly compared with main-sequence stars, but exhibit similar activity levels ${ }^{7}$. We show that the two evolutionary stages fall together in the rotation-activity diagram and form a single sequence in the unsaturated regime in relation only to Rossby numbers derived from stellar models, confirming earlier preliminary results that relied on a more simplistic parametrization of the convective turn-over time ${ }^{8,9}$. This mirrors recent results of fully convective $M$ dwarfs, which likewise fall on the same rotation-activity sequence as partially convective solartype stars ${ }^{10,11}$. Our results demonstrate that turbulence plays a crucial role in driving stellar dynamos and suggest that there is a common turbulence-related dynamo mechanism explaining the magnetic activity of all late-type stars.
\end{abstract}

Activity caused by surface magnetism is a pervasive feature of cool late-type stars, where a dynamo mechanism is supported in the outer convective envelopes of the stellar interiors. The detailed mechanism responsible for this dynamo is still debated, but its basic ingredients include convective turbulence and non-uniform stellar rotation profiles ${ }^{4}$, although the role of the former divides opinions. While it is generally assumed that the levels of stellar activity ${ }^{1,2,12,13}$ and magnetism ${ }^{3,14,15}$ are best explained to scale in relation to the Rossby number, Ro $=P_{\text {rot }} / \tau_{c}$, that is, the ratio between the stellar rotation period, $P_{\text {rot }}$ and the convective turn-over time, $\tau_{c}$, this assumption has also been contested ${ }^{5,6}$. The main point of criticism against the Rossby number has been that the convective turn-over time is not a directly observable quantity and has to be estimated instead using either stellar structure models ${ }^{16}$ or empirical fits ${ }^{12}$, increasing the risk of introducing systematic errors into the analysis. On the main-sequence stars, it has been noted that the coronal X-ray luminosity, $L_{\mathrm{X}}$, can be directly correlated with the rotation period ${ }^{5}$ as $L_{\mathrm{X}} \propto P_{\text {rot }}^{-2}$. Equivalently, the ratio of X-ray to bolometric luminosity, $L_{\mathrm{bol}}$, has been related to the rotation period and stellar radius, $R$, as $L_{\mathrm{X}} / L_{\text {bol }} \propto P_{\text {rot }}^{-2} R^{-4}$, resulting in a marginally better fit than when relating $L_{\mathrm{X}} / L_{\mathrm{bol}}$ to empirical Rossby numbers of the same stars ${ }^{6}$.

Resolving the controversy over whether or not the Rossby number is required for describing the activity scaling is important, as it gives direct clues on some of the key ingredients of the dynamo process that are operational in the stellar convection zones. The two prevailing dynamo paradigms differ mainly by the concept of how poloidal magnetic fields are generated from the toroidal field that arises from the action of differential rotation. In Babcock-Leighton dynamos ${ }^{17,18}$, the poloidal field generation occurs due to thin, rising flux tubes that become twisted by rotation, a process currently assumed to be unrelated to turbulent convection, while in the distributed turbulent dynamo scenario introduced by Parker ${ }^{19}$, the generation of poloidal fields occurs directly by rotationally affected convective cells. A dependence on the Rossby number, describing the rotational influence on convective turbulence, would indicate an important role of turbulence in the dynamo process and a preference to the latter type of dynamo scenario.

Some recent studies have addressed the influence of turbulent convection on rising flux tubes, but have been unable to directly compute the influence on the Babcock-Leighton mechanism ${ }^{20}$. Dedicated global magnetoconvection simulations, in contrast, do show cyclic dynamo action from turbulent convection alone, but do not capture all of the observed properties correctly ${ }^{21,22}$. Although buoyant magnetic structures form in such simulations, they are much larger in scale than the thin, rising flux tubes typically envisaged in Babcock-Leighton models ${ }^{23}$. Hence, continued efforts to develop observational tests are vital.

As stars evolve off the main sequence, they develop increasingly thick convective envelopes, and as a result, their convective turnover time will start to increase ${ }^{16}$. At the same time, the rotation period of these stars will also continue to increase due to magnetic braking ${ }^{24-26}$, leading to diverging evolution trajectories of $P_{\text {rot }}$ and Ro. This offers a possibility to test whether or not the Rossby number offers the best parametrization for the activity scaling. A suitable dataset for this study, containing both main-sequence and evolved stars, is provided by the chromospheric time series collected during the Mount Wilson Observatory (MWO) HK Project ${ }^{27}$. These data allow straightforward derivation of $P_{\text {rot }}$ and the average ratio of the $\mathrm{Ca}$ II $\mathrm{H}$ - and K-line core emission surface flux to bolometric flux, $R_{\mathrm{HK}}^{\prime}=F_{\mathrm{HK}}^{\prime} / F_{\mathrm{bol}}$, which quantifies the efficiency with which the full energy output of a star is converted to chromospheric heating. We then derived the convective turn-over times from stellar structure model ${ }^{28}$ by fitting the model evolutionary tracks to the observed astrophysical parameters of each star. In this way, we were able to estimate the Rossby numbers of both the main-sequence and the evolved stars in a fully uniform way. The Hertzsprung-Russell diagram of the stellar sample is shown in Fig. 1, together with the model tracks and isocontours of the resulting $\tau_{\mathrm{c}}$ values.

In Fig. $2 \mathrm{a}$, we show the rotation-activity relation of $R_{\mathrm{HK}}^{\prime}$ versus Ro. Each star in the figure is coloured by its observed surface gravity, $\log g$, to give an indication of its evolutionary status. Despite a wider scatter of the evolved stars with low surface gravity, it is evident that correlating activity against the Rossby number places them in a common activity sequence with the main-sequence stars.

'Max Planck Institute for Solar System Research, Göttingen, Germany. ${ }^{2}$ Department of Computer Science, Aalto University, Aalto, Finland. ${ }^{3}$ nnstitut für Astrophysik, Georg-August-Universität Göttingen, Göttingen, Germany. $₫ e$-mail: lehtinen@mps.mpg.de 


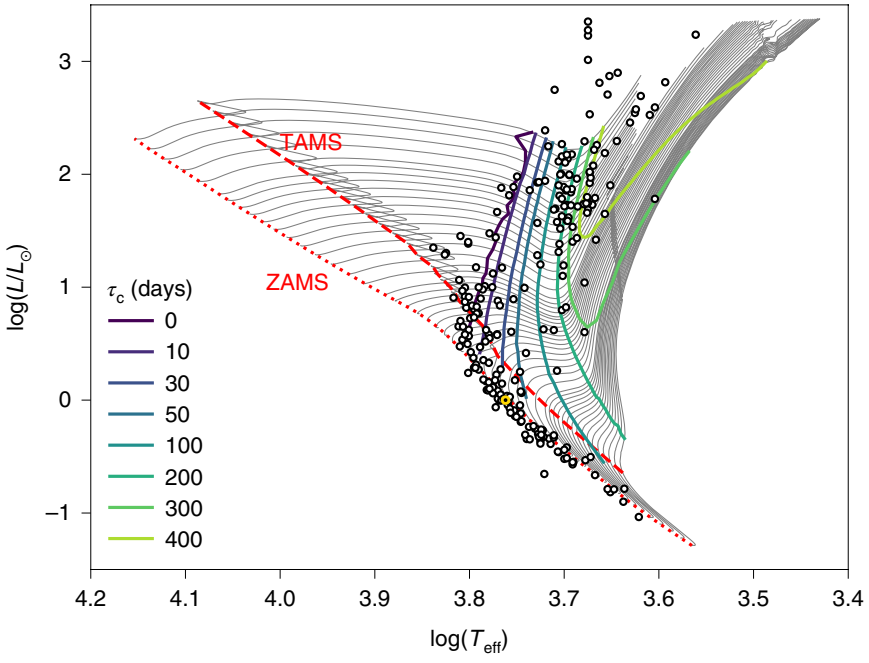

Fig. 1 | Hertzsprung-Russell diagram of the stellar sample. The stars in the sample (black circles), together with a subset of the model evolutionary tracks (thin grey lines) used to determine their convective turn-over times. For clarity, only the solar metallicity tracks are shown here. The red dotted and dashed lines mark the zero-age main sequence (ZAMS) and the termination-age main sequence (TAMS), respectively, while $\tau_{c}$ isocontours are shown as solid coloured lines. The Sun is indicated by the yellow circle.

As a comparison, correlating $R_{\mathrm{HK}}^{\prime}$ directly with $P_{\text {rot }}$ separates the giants into their own distinct group, having similar activity levels to the main-sequence stars, but 10 to 100 times longer rotation periods (see Extended Data Fig. 1).

Scatter remaining in the derived Rossby numbers can be attributed largely to model uncertainties in deriving the $\tau_{\mathrm{c}}$ values from the structure model fits. In Fig. $2 \mathrm{~b}$, we show $R_{\mathrm{HK}}^{\prime}$ versus Ro for each star for which an evolutionary track could be fitted, together with internal error estimates for Ro. Most of the outliers on the large Rossby side of the diagram correspond to the shortest turn-over times $(<5 \mathrm{~d})$, shown as open symbols in the figure. These stars are located in the Hertzsprung-Russell diagram close to the limit where the outer convection zone first appears as intermediate-mass stars (of $\sim 2$ solar masses $\left(M_{\odot}\right)$ ) evolve from the main sequence to the subgiant phase (compare the $\tau_{\mathrm{c}}=0 \mathrm{~d}$ contour in Fig. 1). This limit remains poorly constrained, and thus, the derived thickness of the convection zone near it is highly sensitive to model uncertainties and errors in the stellar parameters. This is in many cases correlated with large internal errors in Ro, although not always, and we have to conclude that the $\tau_{c}$ estimates of these stars are dominated by large systematic errors. Since these systematics are not sufficiently understood, we exclude the stars with $\tau_{\mathrm{c}}<5 \mathrm{~d}$ from our analysis due to their unreliability. The scatter among the remaining stars is related largely to the subgiant phase, as can be expected due to the short evolutionary timescale of the depth of the convection zone during these phases ${ }^{16}$. A handful of outliers are found with both low $R_{\mathrm{HK}}^{\prime}$ values and Rossby numbers. These are most likely stars with spurious $P_{\text {rot }}$ detections due to weak rotational modulation in their activity data.

In contrast, alternative activity scaling relations, $L_{\mathrm{HK}}$ versus $P_{\text {rot }}$, where $L_{\mathrm{HK}}$ is chromospheric Ca II $\mathrm{H}$ and $\mathrm{K}$ luminosity, and $R_{\mathrm{HK}}^{\prime}$ versus $P_{\text {rot }}^{-2} R^{-4}$, which aim to remove the $\tau_{\mathrm{c}}$ dependence from the scaling ${ }^{5,6}$, fail to close the gap between the main-sequence and evolved stars (Fig. 3). This can be formally demonstrated with Gaussian clustering by finding the optimal configuration of bivariate Gaussian distributions to describe the data. For the Rossby scaling, we find a single narrow cluster jointly describing both the main-sequence and evolved stars, with an overlapping cluster corresponding to outliers
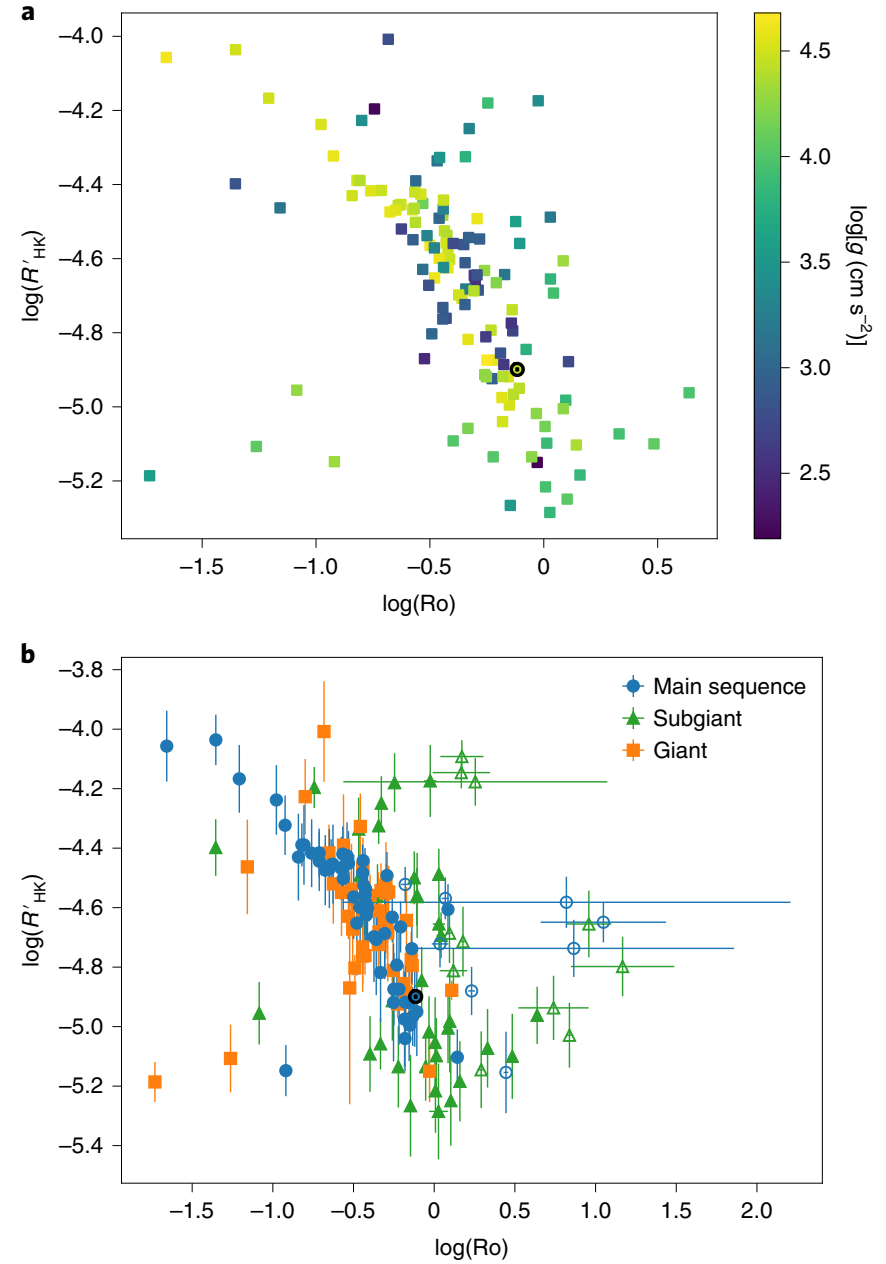

Fig. 2 | Rotation-activity relation for main-sequence and evolved stars. a, Chromospheric $\mathrm{Ca} ॥ \mathrm{H}$ and $\mathrm{K}$ emission ratio versus Rossby number, with the colour scale denoting the stellar surface gravity, decreasing from the main sequence towards giant stars. The position of the Sun is indicated by the circled dot symbol. $\mathbf{b}$, Same as in $\mathbf{a}$, but with error bars showing the internal errors of Ro and the $\pm 3 \sigma$ variability ranges of $R_{H K}^{\prime}$ and distinguishing main-sequence (blue circles), subgiant (green triangles) and giant (orange squares) stars. The shortest turn-over times $(<5 d)$ have large systematic uncertainties associated with them. These stars are shown with open symbols and are excluded from the analysis and $\mathbf{a}$.

(Extended Data Fig. 2a), while for the other two relations, separate clusters are needed for the main-sequence and evolved stars (Extended Data Fig. 2b,c).

Furthermore, investigating the relation of $R_{\mathrm{HK}}^{\prime}$ to a more general quantity of the form $P_{\text {rot }} R^{\alpha}$ reveals that when optimizing for the value of the exponent $\alpha$, to bring the main-sequence and evolved stars together, a coherent scaling relation between activity and the rotation quantity is practically completely lost. Assuming that the scaling with Rossby number holds, we infer that $P_{\text {rot }} / \tau_{\mathrm{c}} \neq P_{\text {rot }} R^{\alpha}$ for any $\alpha$. This demonstrates that $\tau_{\mathrm{c}}$ cannot be adequately described by a single stellar parameter, such as radius, as discussed further in the Supplementary Information.

For the $R_{\mathrm{HK}}^{\prime}$ versus Ro relation, we find residual root-meansquared scatter of RMS $\left(\log \mathrm{R}_{\mathrm{HK}}^{\prime}\right)=0.113 \pm 0.006$ against a linear fit within the main Gaussian cluster. This is smaller than the scatter of $\mathrm{RMS}\left(\log \mathrm{R}_{\mathrm{HK}}^{\prime}\right)=0.21 \pm 0.02$ for the main-sequence stars under the $R_{\mathrm{HK}}^{\prime}$ versus $P_{\text {rot }}^{-2} R^{-4}$ relation. Hence, the model-based Rossby numbers explain better the behaviour of the main-sequence 

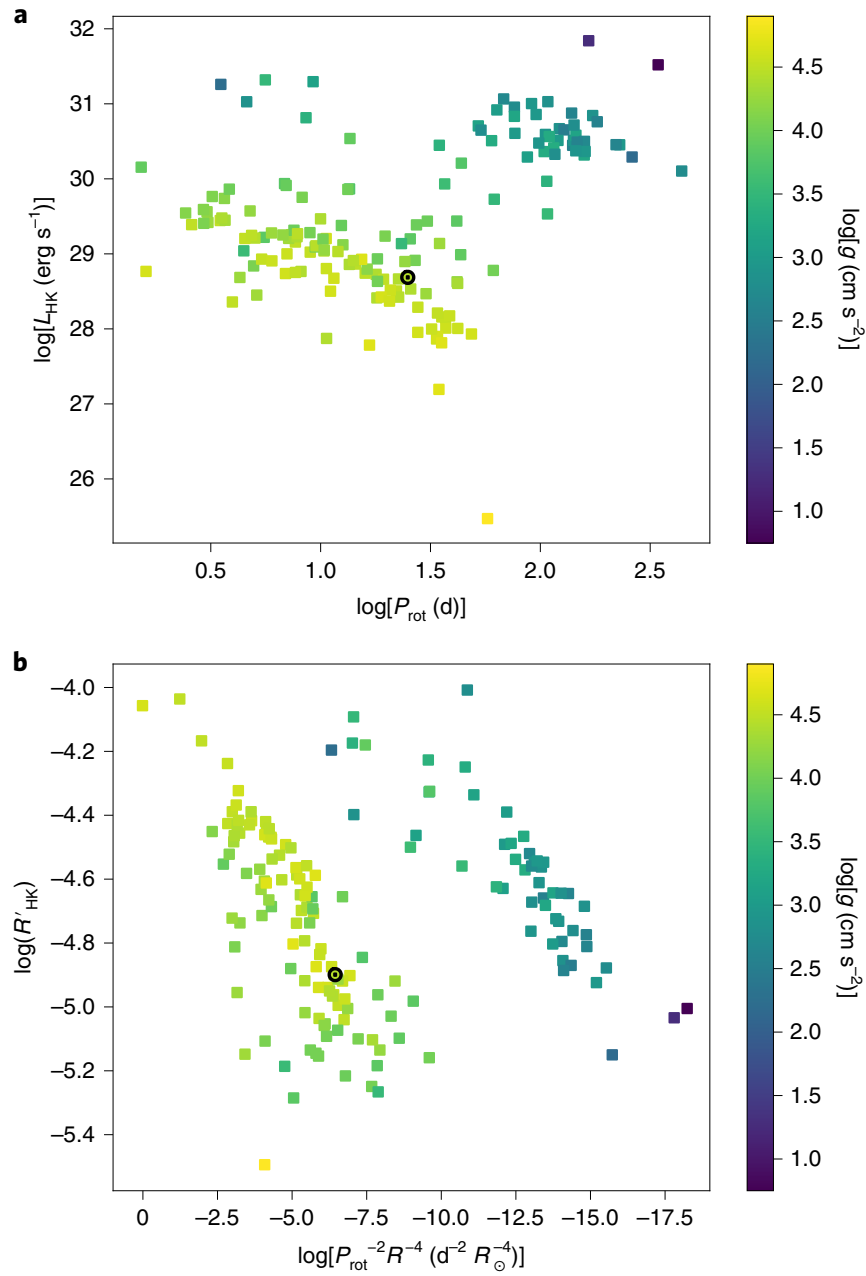

Fig. 3 | Alternative rotation-activity relations independent of convective turn-over time. a, Chromospheric $\mathrm{Ca} ॥ \mathrm{H}$ and $\mathrm{K}$ luminosity versus rotation period. b. Chromospheric $\mathrm{Ca} ॥ \mathrm{H}$ and $\mathrm{K}$ emission ratio versus combined rotation period and stellar radius. The colour scales indicate surface gravity as in Fig. 2a, and the position of the Sun is indicated by the circled dot symbol. $R_{\odot}$, solar radius.

stars, opposite to what was found using X-ray data and empirically determined Rossby numbers 6 . On the evolved stars alone, the larger model uncertainties remain apparent in the residual scatter, yielding $\mathrm{RMS}\left(\log \mathrm{R}_{\mathrm{HK}}^{\prime}\right)=0.17 \pm 0.02$ against Ro, compared with RMS $\left(\log \mathrm{R}_{\mathrm{HK}}^{\prime}\right)=0.12 \pm 0.02$ against $P_{\text {rot }}^{-2} R^{-4}$.

Similar behaviour can be seen by correlating the CaII $\mathrm{H}$ and K surface flux against $P_{\text {rot }}$ and Ro (Extended Data Fig. 3), as was done in some previous works ${ }^{8,9}$. As before, the main-sequence and evolved stars overlap only when correlated with the Rossby number, although now there is no clearly defined activity sequence, as seen for $R_{\mathrm{HK}}^{\prime}$.

The best-fitting power-law model for the Rossby scaling, resulting from the clustering analysis, has the form $R_{\mathrm{HK}}^{\prime} \propto \mathrm{Ro}^{-1.0 \pm 0.1}$. It has a notably shallower slope than what has been reported for X-ray activity ${ }^{5,29}$, canonically $L_{\mathrm{X}} / L_{\mathrm{bol}} \propto \mathrm{Ro}^{-2}$. The cause for this difference is not yet known, but it is most likely related to differences in the relevant emission processes, as X-ray activity is observed to scale up steeply with respect to the Ca II $\mathrm{H}$ and $\mathrm{K}$ emission ${ }^{30}$. Our scaling relation also does not capture the saturated activity regime at low Rossby numbers where the activity scaling of main-sequence stars is observed to flatten and lose its rotation dependence $5,6,1,29$. This is due to the limited Rossby range in our stellar sample, which does not extend to sufficiently low Rossby numbers. Thus, we cannot, at this stage, explicitly confirm whether or not the evolved stars follow the same activity saturation as the main-sequence stars. Some of the fastest-rotating superactive giants found in synchronously rotating binary systems may yet provide test cases for the presence of the saturation regime in evolved stars.

These results constitute a strong argument that at least in the rotation-dependent regime at higher Rossby numbers, Ro provides both a necessary and sufficient parametrization for the stellar activity level over a wide range of evolutionary stages. This confirms findings from earlier works ${ }^{8,9}$ that assumed a simplistic parametrization of the convective turn-over time as a function of the effective temperature for all evolved stars. The Rossby-independent parametrizations for the rotation-activity relation break down once evolved stars are considered. The fact that a common scaling independent of the evolutionary stage can be achieved only when both stellar rotation and convection are taken into account suggests that the underlying dynamos operating in these stars follow the turbulent dynamo paradigm. Since fully convective M dwarfs have been shown to follow the same activity scaling against the Rossby number as more massive partially convective main-sequence stars ${ }^{10,11,29}$, it is reasonable to assume that all late-type stars share the same fundamental dynamo mechanism irrespective of their mass or evolutionary stage and their resulting, vastly different internal structures.

\section{Methods}

Chromospheric activity and astrophysical parameters. The initial sample selection consists of all of the stars included in the MWO HK Project ${ }^{27}$ that have time-series observations spanning over five years and covering at least four complete observing seasons. This selection consists of 224 stars in total. The majority of the stellar sample consists of single stars, and the remaining binary members all have wide enough orbits so that none of them has tidally synchronized rotation. Using sufficiently extended time-series data ensures averaging over yearly activity variations and facilitates a more reliable rotation-period search. The final sample for which it was possible to determine both $P_{\text {rot }}$ and $\tau_{c}$ consists of 58 mainsequence and 92 evolved stars. We supplemented this sample by five moderately to very active main-sequence stars that have a poorer coverage in the MWO data but have accurate $P_{\text {rot }}$ values available from photometric studies ${ }^{31}$.

The Ca II $\mathrm{H}$ and $\mathrm{K}$ emission ratios, $R_{\mathrm{HK}}^{\prime}=L_{\mathrm{HK}} / L_{\mathrm{bol}}=F_{\mathrm{HK}}^{\prime} / \sigma T_{\text {eff }}^{4}$, where $T_{\text {eff }}$ is the effective temperature, were calculated from the averaged MWO S-index observation $\mathrm{s}^{32}$ after removing sections of data with apparent calibration issues and outliers more than $4 \sigma$ away from the sample mean ${ }^{33}$. The conversion is colour dependent and defined separately for the main-sequence and evolved stars ${ }^{34}$. The choice of appropriate conversion law was made based on the absolute V-band magnitude, $M_{\mathrm{V}}$, so that stars with $M_{\mathrm{V}}$ values more than 1 mag above the main sequence ${ }^{35}$ were treated as evolved stars, and the rest as main-sequence stars. For the cooler stars with $B-V \geq 0.8 \mathrm{mag}$ (or $T_{\text {eff }} \leq 5,300 \mathrm{~K}$ ), this procedure neatly separates the main-sequence from the evolved stars (see Fig. 1). For the hotter stars, there is no clear separation between the different evolutionary stages in the Hertzsprung-Russell diagram, but this causes no issues for calculating $R_{\mathrm{HK}}^{\prime}$, since the two conversion laws overlap in this region ${ }^{34}$. Due to the length of the activity time series, the internal errors of the mean activity levels are extremely small, being of the order of $\sigma=0.0001$ or less for both the $\mathrm{S}$ index and $\log \mathrm{R}_{\mathrm{HK}}^{\prime}$. Note that the vertical error bars in Fig. 2b indicate the $\pm 3 \sigma$ variability ranges of $R_{\mathrm{HK}}^{\prime}$ and are thus much wider than the internal errors.

$V$-band magnitudes and $B-V$ colours (Vega magnitude system) were adopted from the Hipparcos photometry ${ }^{36}$ for all stars apart from the Sun ${ }^{35}$ Parallaxes were drawn from Gaia Data Release $2^{37-39}$, where available, otherwise adopting Hipparcos parallaxes ${ }^{40}$. Interstellar extinction in the $\mathrm{V}$ band was assumed to be $a_{\mathrm{V}}=1.5 \mathrm{mag} \mathrm{kpc}^{-1}$ for calculating the absolute magnitudes ${ }^{41}$, which is a workable assumption since the stellar sample is located largely in our local galactic neighbourhood and contains field stars located only outside regions with high extinction.

We adopted literature values for the $T_{\text {eff }}$ luminosity ${ }^{35,42}, L$, and metallicity ${ }^{43}$, $[\mathrm{Fe} / \mathrm{H}]$, of the stars. For some stars, no values of $T_{\text {eff }}$ or $L$ were available, and these had to be estimated from the photometry $y^{44}$. Values of $R$ were, likewise, estimated for all stars on the basis of the photometry and effective temperatures ${ }^{45}$. For all except the most distant stars in our sample, we estimate the errors of the derived radii to be dominated by the errors in $T_{\text {eff }}$ (assuming uncertainties of $100 \mathrm{~K}$ ), leading to a representative error level in $R$ that is below $10 \%$. The Ca II $\mathrm{H}$ and K luminosities were calculated from the emission fluxes as $L_{\mathrm{HK}}=4 \pi R^{2} F_{\mathrm{HK}}^{\prime}$. Values of logg were compiled from primary sources through the PASTEL (PAramètres STELlaires) catalogue ${ }^{46}$. The observationally estimated log $g$ values can have substantial uncertainties associated with them ${ }^{47}$. These are, however, not 
critical to the present study, as we have used $\log g$ only as an observational indicator for the stellar evolutionary stage and have not used its values for any further calculations.

Stellar structure models. Since $\tau_{\mathrm{c}}$ depends on the stellar mass, chemical composition and evolutionary stage, we derived its value for each star individually using a grid of stellar evolution models from the Yale-Potsdam Stellar Isochrones ${ }^{28}$ (YaPSI). In the YaPSI models, $\tau_{\mathrm{c}}$ is calculated according to a global definition ${ }^{48}$ based on an average over the whole convection zone of the star.

The position of each star in the Hertzsprung-Russell diagram (that is, $\log T_{\text {eff }}$ and $\log \left(L / L_{\odot}\right)$, where $L_{\odot}$ is solar luminosity) was compared with the model evolutionary tracks, and a subset of tracks matching its parameters within the observational uncertainties was selected. We assumed uncertainties of $100 \mathrm{~K}$ in $T_{\text {eff }}$ and $0.12 \mathrm{dex}$ (units of decimal exponent) in $\log \left(L / L_{\odot}\right)$. A best-fitting track was then generated from the selected tracks by linear interpolation, and $\tau_{c}$ was extracted from this synthetic track at the point of closest approach to the observed parameters. We repeated this procedure for three different metallicities $-[\mathrm{Fe} / \mathrm{H}]=-0.5,0.0$ and 0.3 (with the initial helium fraction kept constant at $Y=0.28$ ) - obtaining an estimate of $\tau_{c}$ in each case. This range of $[\mathrm{Fe} / \mathrm{H}]$ encompasses almost the totality of the stars within our sample (Extended Data Fig. 4). The final value of $\tau_{c}$ was obtained by linear interpolation in $[\mathrm{Fe} / \mathrm{H}]$. The range of $\tau_{\mathrm{c}}$ obtained from the models at different metallicities was used to estimate the uncertainty in $\tau$. It should be noted that although this uncertainty is only qualitative (that is, it cannot be interpreted as a formal error bar in $\tau_{\mathrm{c}}$ ) and it underestimates the uncertainty due to errors in $T_{\text {eff }}$ and $L / L_{\odot}$, we found that the stars with the largest uncertainty are those close to the $\tau_{c} \approx 0 \mathrm{~d}$ limit, lending further support to excluding the stars with the smallest $\tau_{\mathrm{c}}$ values from the analysis. The uncertainties of Ro, as shown in Fig. 2b, were computed by propagation of uncertainty from the $\tau_{\mathrm{c}}$ uncertainties and the $P_{\text {rot }}$ error estimates.

For stars on the main sequence, our theoretical values of $\tau_{\mathrm{c}}$ are in good qualitative agreement with the classical empirical estimates ${ }^{12}, \tau_{c, \text { empirical }}$, except for a factor between the two of $\tau_{\mathrm{c}} / \tau_{\mathrm{c} \text {,empirical }} \approx 2.6$. For stars in the subgiant and red giant branch phases, the stellar evolution models predict a strong dependence of $\tau_{c}$ on the evolutionary stage of the star, which is not captured by the empirical estimates.

The evolutionary stages of the stars, indicated in Fig. 2b, were determined from the evolutionary track fits using the following criteria. The termination-age main sequence occurs when the hydrogen mass fraction in the core of a star reaches below $10^{-4}$, after which the stars enter their subgiant phase. The transition from the subgian to the giant phase was set to occur at the bottom of the red giant branch, which was defined to be reached once the inert helium core reaches a mass of $M_{\mathrm{He}}>0.1 M_{\odot}$.

Determining precise stellar properties for red giants by matching their observed properties to evolutionary tracks and isochrones is notoriously difficult ${ }^{49,50}$. In addition, a substantial source of scatter in the value of $\tau_{c}$ for subgiant and early red giant stars comes from their fast evolutionary timescales and is therefore, unfortunately, unavoidable. This effect is illustrated in Extended Data Fig. 5, which shows a selection of evolution tracks for the main-sequence and postmain-sequence phases and the corresponding evolution of $\tau_{\mathrm{c}}$. For stars of mass $M<1.3 M_{\odot}, \tau_{\mathrm{c}}$ remains essentially constant during the main sequence and increases smoothly during the post-main-sequence evolution. In contrast, stars of $M>1.5 M_{\odot}$ have no outer convection zone in the main sequence. As they reach the bottom of the red giant branch, a sizeable convective envelope develops on a much faster timescale than in their less massive counterparts. Correspondingly, $\tau_{c}$ changes very rapidly from zero to a few hundred days, a value typical of red giant stars.

Period analysis. The rotation periods were determined for the stars from the rotational modulation in the MWO S-index time series, caused by the transit of chromospheric active regions over the visible stellar hemispheres ${ }^{33}$. An initial period search was performed for each complete observing season, using periodic Gaussian processes ${ }^{51}$. If the different seasons yielded repeatedly comparable period values, these were used as initial guesses for computing the final period estimate from the full time series using the continuous period search method ${ }^{52}$, comprising harmonic fits performed using a sliding window. The full period analysis provided both time-averaged values and error estimates for $P_{\text {rot }}$, where the error estimates were derived as the standard error of the mean of a mutually independent sample of individual $P_{\text {rot }}$ estimates over the duration of the time series. Finally, the solar $P_{\text {rot }}$ estimate was rescaled from the synodic frame, corotating with Earth's orbital motion, to the sidereal frame to be directly comparable with the stellar rotation periods.

Four stars with low activity levels received unexpectedly small $P_{\text {rot }}$ values from the period analysis (Fig. 2b lower left corner). The stars in question are HD 3443 , HD 3795, HD 22071 and HD 190360. Each of these stars has a low activity level, and it is possible that the rotation modulation signal in their activity time series is masked by observational errors. The period analysis results for these stars show poor stability in time and lead to periods of $\sim 5 \mathrm{~d}$, which could be related to an effective sampling frequency in the time series. The most likely explanation for these small $P_{\text {rot }}$ values, therefore, is that they are spurious period detections.

Many of the stars have previous $P_{\text {rot }}$ estimates available from the literature. We found that our estimates generally coincide with these values, although especially in the case of several slowly rotating giants, the previously available $P_{\text {rot }}$ values appear to be fairly inaccurate. For three stars (HD 47442, HD 111456 and HD 158614), we were unable to reproduce a previously reported period detection and thus left these stars' periods undetermined. A full comparison table between our $P_{\text {rot }}$ estimates and the literature periods can be found online at the Strasbourg Astronomical Data Center, together with the rest of our numerical results.

Gaussian clustering. The clustering analysis of the rotation-activity diagrams was performed using a Gaussian mixture model with expectation-maximization ${ }^{53}$. We searched for the statistically most likely configuration of clusters, not assuming any previous knowledge about either the number of clusters or their covariances. The algorithm was run for numbers of clusters ranging from one to five, and the optimal configuration was determined by minimizing the Bayesian information criterion $^{54}$. The most probable cluster membership was determined afterwards for the individual stars, using the Mahalanobis distance ${ }^{55}$.

We determined the slope of the empirical rotation-activity scaling relation by linear least-squares fitting to stars identified as members of a single Gaussian cluster. The residual scatter reported for the $R_{\mathrm{HK}}^{\prime}$ versus Ro and $R_{\mathrm{HK}}^{\prime}$ versus $P_{\mathrm{rot}}^{-2} R^{-4}$ scaling relations was calculated against these fits, with error estimates derived by bootstrapping the fit residuals.

\section{Data availability}

The MWO HK Project data are available online at ftp://solis.nso.edu/ MountWilson_HK/, and Gaia Data Release 2 from the Gaia Archive at http:// gea.esac.esa.int/archive/. The YaPSI stellar models are available at http://www. astro.yale.edu/yapsi/. The adopted and derived astrophysical parameters for the stellar sample used in this study are available in online tables at the Strasbourg Astronomical Data Center via anonymous ftp to cdsarc.u-strasbg.fr (130.79.128.5) or via http://cdsarc.u-strasbg.fr/viz-bin/qcat?J/other/NatAs.

Received: 8 July 2019; Accepted: 6 February 2020; Published online: 09 March 2020

\section{References}

1. Mamajek, E. E. \& Hillenbrand, L. A. Improved age estimation for solar-type dwarfs using activity-rotation diagnostics. Astrophys. J. 687, 1264-1293 (2008)

2. Wright, N. J., Drake, J. J., Mamajek, E. E. \& Henry, G. W. The stellar-activityrotation relationship and the evolution of stellar dynamos. Astrophys. J. 743, 48 (2011)

3. Reiners, A., Basri, G. \& Browning, M. Evidence for magnetic flux saturation in rapidly rotating M stars. Astrophys. J. 692, 538-545 (2009).

4. Charbonneau, P. Dynamo models of the solar cycle. Living Rev. Sol. Phys. 7, 3 (2010).

5. Pizzolato, N., Maggio, A., Micela, G., Sciortino, S. \& Ventura, P. The stellar activity-rotation relationship revisited: dependence of saturated and non-saturated X-ray emission regimes on stellar mass for late-type dwarfs. Astron. Astrophys. 397, 147-157 (2003).

6. Reiners, A., Schüssler, M. \& Passegger, V. M. Generalized investigation of the rotation-activity relation: favoring rotation period instead of Rossby number. Astrophys. J. 794, 144 (2014).

7. Strassmeier, K. G., Fekel, F. C., Bopp, B. W., Dempsey, R. C. \& Henry, G. W. Chromospheric Ca II $\mathrm{H}$ and $\mathrm{K}$ and $\mathrm{H}$-alpha emission in single and binary stars of spectral types F6-M2. Astrophys. J. Suppl. Ser. 72, 191-230 (1990).

8. Basri, G. Stellar activity in synchronized binaries. II. A correlation analysis with single stars. Astrophys. J. 316, 377-388 (1987).

9. Young, A., Ajir, F. \& Thurman, G. Chromospheric activity in evolved stars: the rotation-activity connection and the binary-single dichotomy. Publ. Astron. Soc. Pac. 101, 1017-1031 (1989).

10. Wright, N. J. \& Drake, J. J. Solar-type dynamo behaviour in fully convective stars without a tachocline. Nature 535, 526-528 (2016).

11. Newton, E. R. et al. The H $\alpha$ emission of nearby $M$ dwarfs and its relation to stellar rotation. Astrophys. J. 834, 85 (2017).

12. Noyes, R. W., Hartmann, L. W., Baliunas, S. L., Duncan, D. K. \& Vaughan, A $\mathrm{H}$. Rotation, convection, and magnetic activity in lower main-sequence stars. Astrophys. J. 279, 763-777 (1984).

13. Gunn, A. G., Mitrou, C. K. \& Doyle, J. G. On the rotation-activity correlation for active binary stars. Mon. Not. R. Astron. Soc. 296, 150-164 (1998).

14. Vidotto, A. A. et al. Stellar magnetism: empirical trends with age and rotation. Mon. Not. R. Astron. Soc. 441, 2361-2374 (2014).

15. Aurière, $M$. et al. The magnetic fields at the surface of active single G-K giants. Astron. Astrophys. 574, A90 (2015).

16. Gilliland, R. L. The relation of chromospheric activity to convection, rotation, and evolution off the main sequence. Astrophys. J. 299, 286-294 (1985).

17. Babcock, H. W. The topology of the Sun's magnetic field and the 22-year cycle. Astrophys. J. 133, 572-587 (1961).

18. Leighton, R. B. A magneto-kinematic model of the solar cycle. Astrophys. J. 156, 1-26 (1969).

19. Parker, E. N. The formation of sunspots from the solar toroidal field. Astrophys. J. 121, 491-507 (1955). 
20. Weber, M. A., Fan, Y. \& Miesch, M. S. The rise of active region flux tubes in the turbulent solar convective envelope. Astrophys. J. 741, 11 (2011).

21. Käpylä, P. J., Mantere, M. J. \& Brandenburg, A. Cyclic magnetic activity due to turbulent convection in spherical wedge geometry. Astrophys. J. Lett. 755 L22 (2012)

22. Augustson, K., Brun, A. S., Miesch, M. \& Toomre, J. Grand minima and equatorward propagation in a cycling stellar convective dynamo. Astrophys. J. 809, 149 (2015).

23. Nelson, N. J., Brown, B. P., Brun, A. S., Miesch, M. S. \& Toomre, J. Buoyant magnetic loops generated by global convective dynamo action. Solar Phys. 289, 441-458 (2014)

24. Skumanich, A. Time scales for Ca II emission decay, rotational braking, and lithium depletion. Astrophys. J. 171, 565-567 (1972).

25. Spada, F., Gellert, M., Arlt, R. \& Deheuvels, S. Angular momentum transport efficiency in post-main sequence low-mass stars. Astron. Astrophys. 589, A23 (2016).

26. Ceillier, T. et al. Surface rotation of Kepler red giant stars. Astron. Astrophys 605, A111 (2017).

27. Wilson, O. C. Chromospheric variations in main-sequence stars. Astrophys. J. 226, 379-396 (1978).

28. Spada, F., Demarque, P., Kim, Y.-C., Boyajian, T. S. \& Brewer, J. M. The Yale-Potsdam Stellar Isochrones. Astrophys. J. 838, 161 (2017).

29. Wright, N. J., Newton, E. R., Williams, P. K. G., Drake, J. J. \& Yadav, R. K. The stellar rotation-activity relationship in fully convective $\mathrm{M}$ dwarfs. Mon. Not. R. Astron. Soc. 479, 2351-2360 (2018).

30. Mittag, M., Schmitt, J. H. M. M. \& Schröder, K. P. Revisiting the connection between magnetic activity, rotation period, and convective turnover time for main-sequence stars. Astron. Astrophys. 618, A48 (2018).

31. Lehtinen, J., Jetsu, L., Hackman, T., Kajatkari, P. \& Henry, G. W. Activity trends in young solar-type stars. Astron. Astrophys. 588, A38 (2016).

32. Egeland, R. et al. The Mount Wilson Observatory S-index of the Sun. Astrophys. J. 835, 25 (2017).

33. Olspert, N., Lehtinen, J. J., Käpylä, M. J., Pelt, J. \& Grigorievskiy, A. Estimating activity cycles with probabilistic methods. II. The Mount Wilson Ca H\&K data. Astron. Astrophys. 619, A6 (2018).

34. Rutten, R. G. M. Magnetic structure in cool stars. VII. Absolute surface flux in Ca II H and K line cores. Astron. Astrophys. 130, 353-360 (1984).

35. Cox, A. N. (ed.) Allen's Astrophysical Quantities 4th edn (Springer, 2000).

36. Battrick, B. (ed.) The Hipparcos and Tycho Catalogues: Astrometric and Photometric Star Catalogues Derived from the ESA Hipparcos Space Astrometry Mission Special Publication No. SP-1200 (ESA, 1997).

37. Gaia Collaboration et al. The Gaia mission. Astron. Astrophys. 595, A1 (2016)

38. Gaia Collaboration et al. Gaia Data Release 2: summary of the contents and survey properties. Astron. Astrophys. 616, A1 (2018).

39. Luri, X. et al. Gaia Data Release 2: using Gaia parallaxes. Astron. Astrophys. 616, A9 (2018).

40. van Leeuwen, F. Validation of the new Hipparcos reduction. Astron. Astrophys. 474, 653-664 (2007)

41. Vergely, J. L., Egret, D., Freire Ferrero, R., Valette, B. \& Koeppen, J. The Extinction in the Solar Neighbourhood from the HIPPARCOS Data in Hipparcos: Venice '97 Special Publication No. SP-402 (eds Perryman, M. A. C. et al.) 603-606 (ESA, 1997).

42. Andrae, R. et al. Gaia Data Release 2: first stellar parameters from Apsis. Astron. Astrophys. 616, A8 (2018).

43. Gáspár, A., Rieke, G. H. \& Ballering, N. The correlation between metallicity and debris disk mass. Astrophys. J. 826, 171 (2016).

44. Flower, P. J. Transformations from theoretical Hertzsprung-Russell diagrams to color-magnitude diagrams: effective temperatures, $B-V$ colors, and bolometric corrections. Astrophys. J. 469, 355-365 (1996).

45. Gray, D. F. The Observation and Analysis of Stellar Photospheres (Cambridge Univ. Press, 2005).

46. Soubiran, C., LeCampion, J.-F., Brouillet, N. \& Chemin, L. The PASTEL catalogue: 2016 version. Astron. Astrophys. 591, A118 (2016).

47. Bastien, F. A., Stassun, K. G., Basri, G. \& Pepper, J. An observational correlation between stellar brightness variations and surface gravity. Nature 500, 427-430 (2013)
48. Kim, Y.-C. \& Demarque, P. The theoretical calculation of the Rossby number and the 'nonlocal' convective overturn time for pre-main-sequence and early post-main-sequence stars. Astrophys. J. 457, 340-347 (1996).

49. Serenelli, A. M., Bergemann, M., Ruchti, G. \& Casagrande, L. Bayesian analysis of ages, masses and distances to cool stars with non-LTE spectroscopic parameters. Mon. Not. R. Astron. Soc. 429, 3645-3657 (2013).

50. Silva Aguirre, V. \& Serenelli, A. M. Asteroseismic age determination for dwarfs and giants. Astron. Nachr. 337, 823-826 (2016)

51. Wang, Y., Khardon, R. \& Protopapas, P. Nonparametric Bayesian estimation of periodic light curves. Astrophys. J. 756, 67 (2012).

52. Lehtinen, J., Jetsu, L., Hackman, T., Kajatkari, P. \& Henry, G. W. The continuous period search method and its application to the young solar analogue HD 116956. Astron. Astrophys. 527, A136 (2011)

53. Barber, D. Bayesian Reasoning and Machine Learning (Cambridge Univ. Press, 2012).

54. Stoica, P. \& Selen, Y. Model-order selection: a review of information criterion rules. IEEE Signal Process. Mag. 21, 36-47 (2004).

55. Mahalanobis, P. C. On the generalized distance in statistics. Proc. Natl Inst. Sci. India 2, 49-55 (1936).

\section{Acknowledgements}

This work has made use of data from the European Space Agency (ESA) mission Gaia (https://www.cosmos.esa.int/gaia), processed by the Gaia Data Processing and Analysis Consortium (DPAC, https://www.cosmos.esa.int/web/gaia/dpac/consortium). Funding for DPAC has been provided by national institutions, in particular the institutions participating in the Gaia Multilateral Agreement. The chromospheric activity data derive from the Mount Wilson Observatory HK Project, which was supported by both public and private funds through the Carnegie Observatories, the Mount Wilson Institute and the Harvard-Smithsonian Center for Astrophysics, starting in 1966 and continuing for over 36 years. These data are the result of work by $\mathrm{O}$. Wilson, A. Vaughan, G. Preston, D. Duncan, S. Baliunas and others. The work has made use of the SIMBAD database at CDS, Strasbourg, France, and NASA's Astrophysics Data System (ADS) services. J.J.L. acknowledges financial support from the Independent Max Planck Research Group 'SOLSTAR'. F.S. acknowledges the support of the German space agency (Deutsches Zentrum für Luft- und Raumfahrt) under PLATO Data Center grant 50OO1501. M.J.K., N.O. and P.J.K. acknowledge the support of the Academy of Finland ReSoLVE Centre of Excellence (grant no. 307411). P.J.K. acknowledges support from DFG Heisenberg (grant no. KA 4825/2-1). This project has received funding from the European Research Council (ERC) under the European Union's Horizon 2020 research and innovation programme (Project UniSDyn, grant agreement no. 818665)

\section{Author contributions}

All authors contributed to the research and its design. J.J.L. and N.O. led the data analysis of the observations. F.S. led the stellar structure modelling. M.J.K. and P.J.K. led the theoretical interpretation of the obtained results. All authors contributed to the discussion of the results and to the manuscript.

\section{Competing interests}

The authors declare no competing interests.

\section{Additional information}

Extended data is available for this paper at https://doi.org/10.1038/s41550-020-1039-x.

Supplementary information is available for this paper at https://doi.org/10.1038/ s41550-020-1039-x.

Correspondence and requests for materials should be addressed to J.J.L.

Peer review information Nature Astronomy thanks Gopal Hazra and the other, anonymous, reviewer(s) for their contribution to the peer review of this work.

Reprints and permissions information is available at www.nature.com/reprints.

Publisher's note Springer Nature remains neutral with regard to jurisdictional claims in published maps and institutional affiliations.

(c) The Author(s), under exclusive licence to Springer Nature Limited 2020 


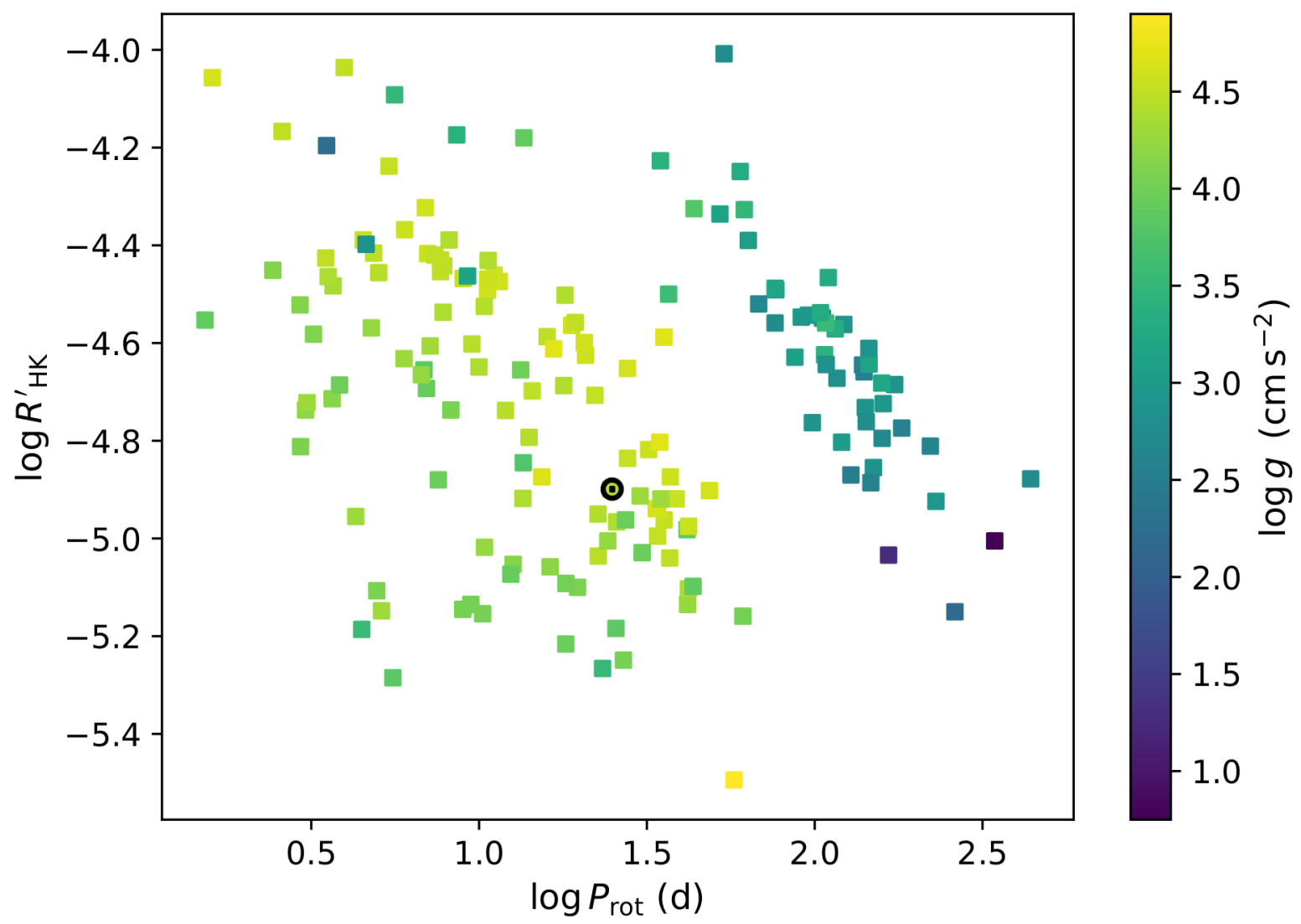

Extended Data Fig. 1 | Chromospheric Ca II H\&K emission ratio, $\boldsymbol{R}_{\mathrm{HK}}^{\prime}$ against the stellar rotation period $\boldsymbol{P}_{\text {rot* }}$ The diagram shows giant stars as a distinct population with comparable activity levels but notably longer rotation periods than main sequence stars, in contrast to the common rotation-activity sequence seen using the Rossby number. The colour scale indicates surface gravity, log $g$, as in Fig. 2a and the position of the Sun is indicated by the circled dot symbol. 
a

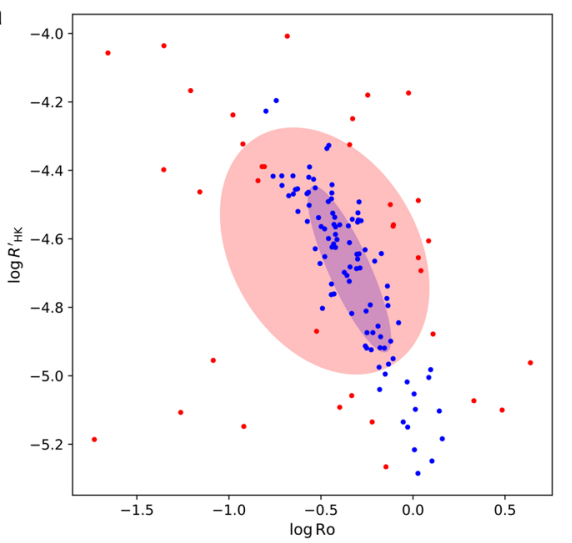

b

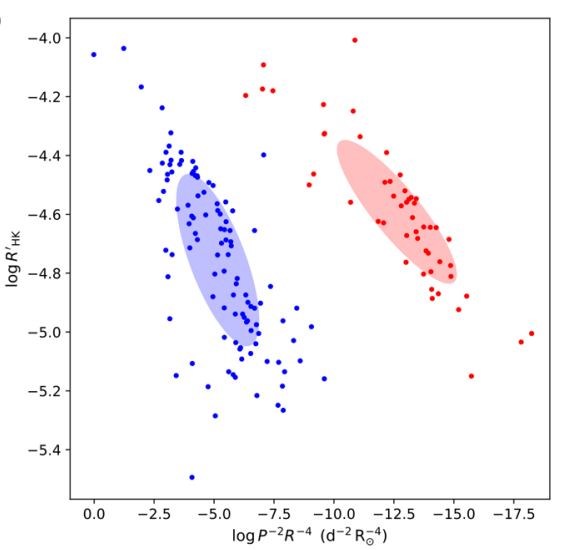

C

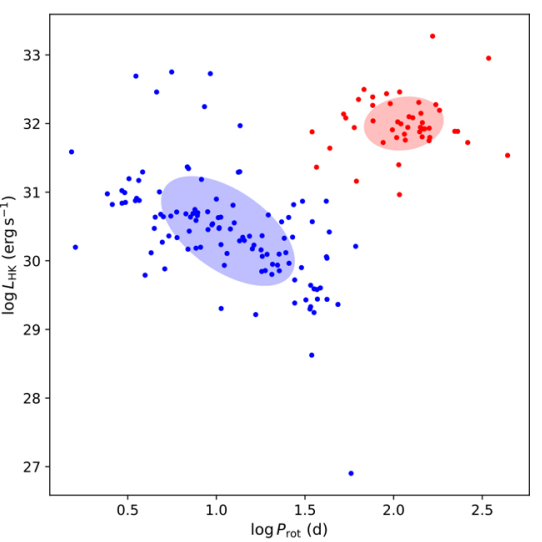

Extended Data Fig. 2 | Gaussian clustering for the rotation-activity relation. a, Chromospheric Ca II H\&K emission ratio, $R_{H K}^{\prime}$, vs. Rossby number, Ro. b, Chromospheric Ca II H\&K emission ratio, $R_{H K}^{\prime}$, vs. combined rotation period and stellar radius, $P_{\text {rot }}^{-2} R^{-4}$. c, Chromospheric Ca II H\&K luminosity $L_{H K}$ vs. rotation period, $P_{\text {rot }}$. Optimal clustering of the data is indicated by the blue and red ellipses, reflecting the corresponding $95 \%$ confidence regions, and individual stars are coloured according to their inferred cluster membership. 
a

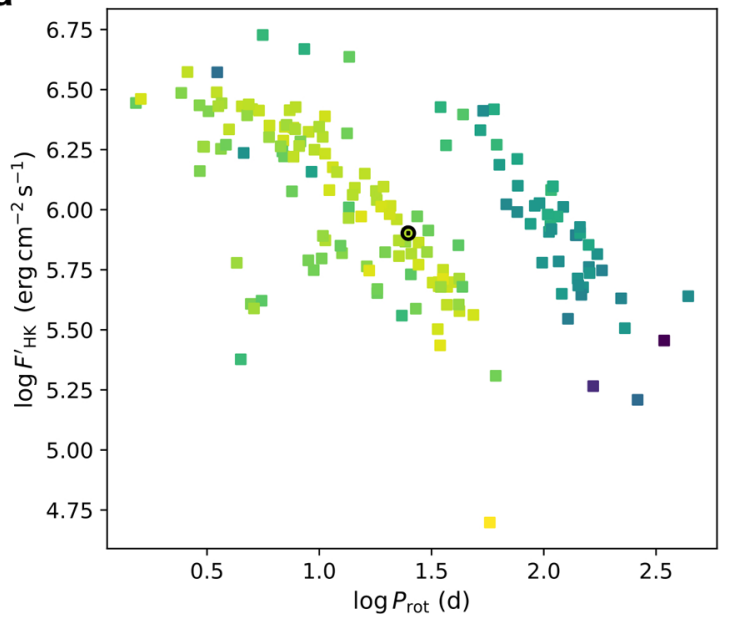

b

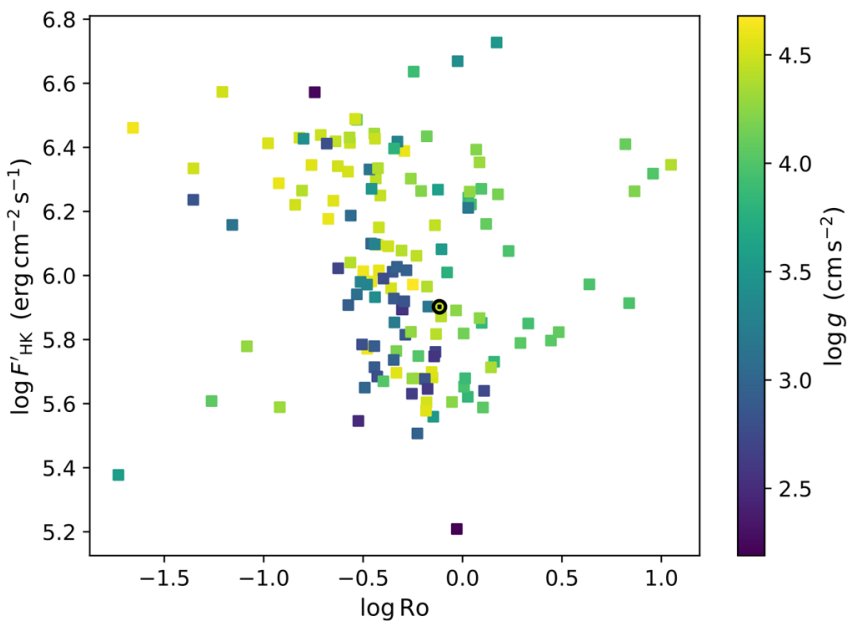

Extended Data Fig. 3 | Rotation-activity relations using the chromospheric Ca II H\&K surface flux $\boldsymbol{F}_{\mathrm{HK}}^{\prime}$. $\mathbf{a}$, Chromospheric surface emission flux, $F_{\mathrm{HK}}^{\prime}$, vs. stellar rotation period, $P_{\text {rot }}$. b. Chromospheric surface emission flux, $F_{H K}^{\prime}$, vs. Rossby number, Ro. The colour scale indicates surface gravity, log $g$, as in Fig. $2 \mathrm{a}$ and the position of the Sun is indicated by the circled dot symbol. 


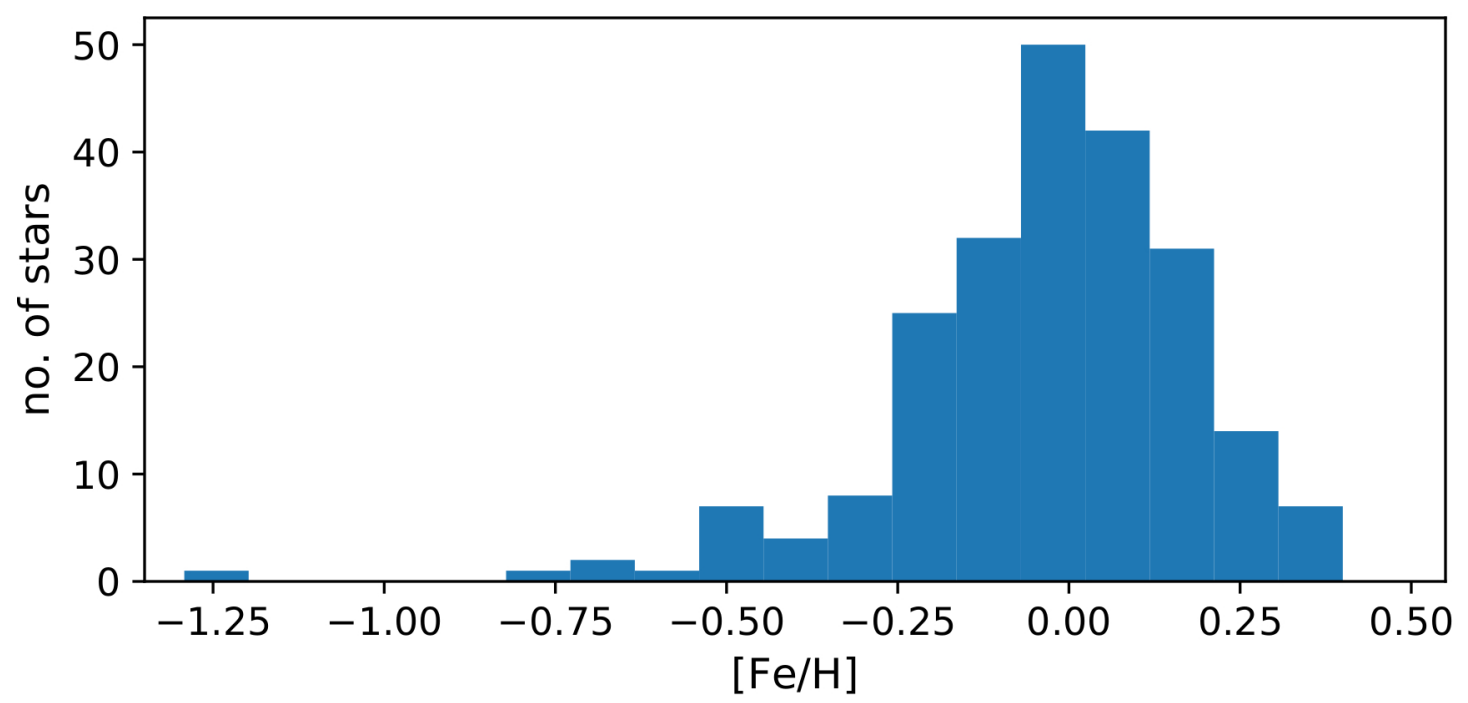

Extended Data Fig. 4 | Metallicity distribution of the sample stars. One low metallicity giant, HD 122563, with [Fe/H] $=-2.42$ is left outside the shown metallicity range since it lacks both a determined $P_{\text {rot }}$ and $\tau_{c}$ value and so does not enter our analysis. 
a

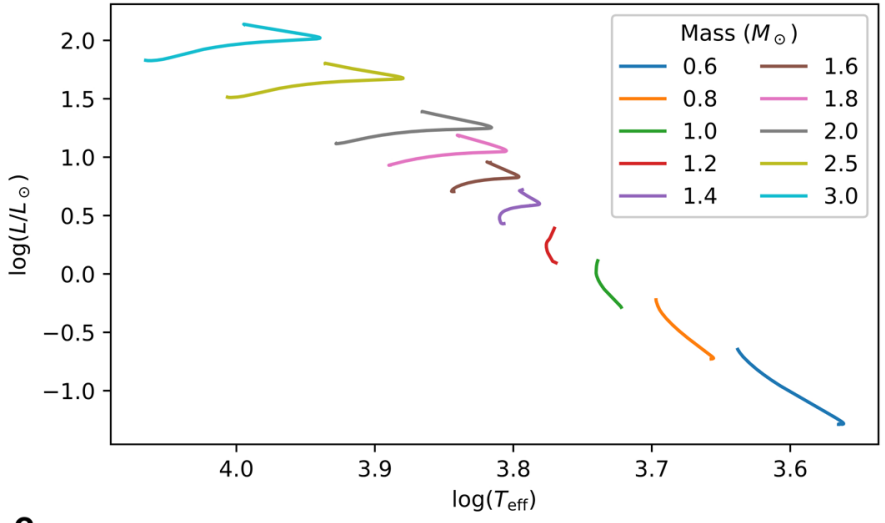

C

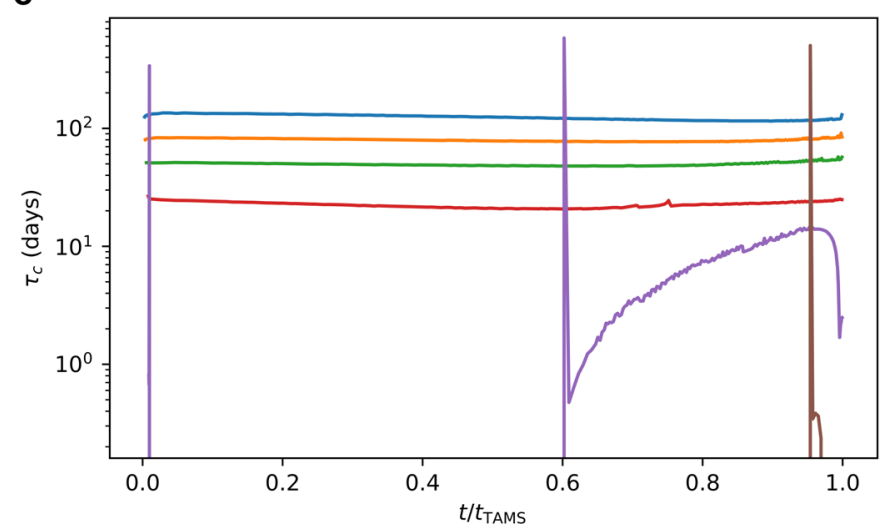

b

TAMS to RGB tip

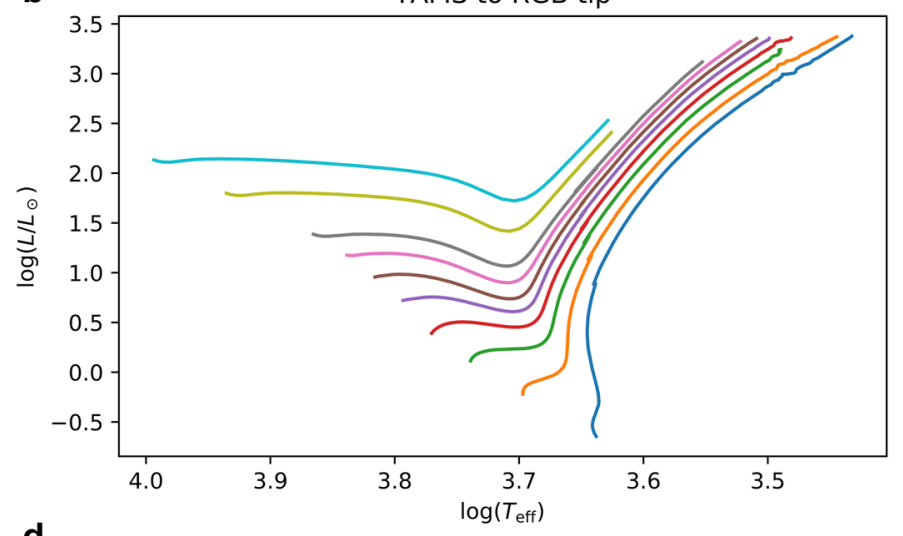

d

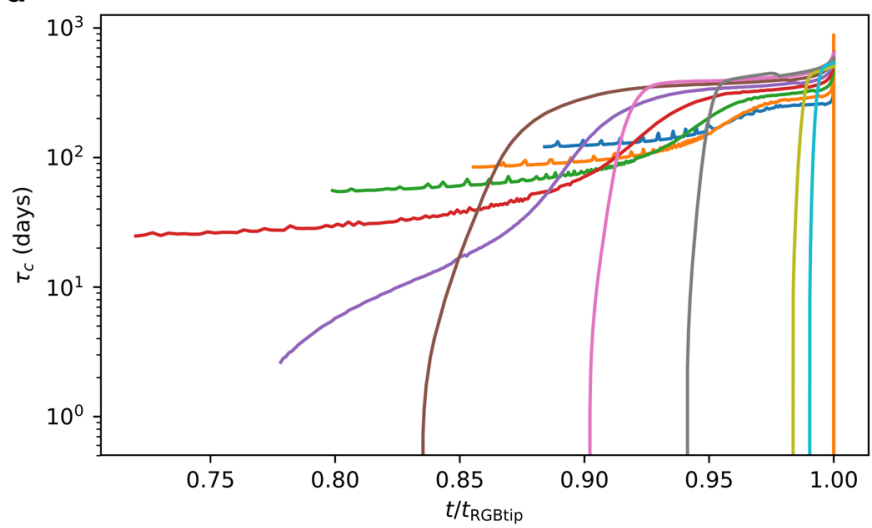

Extended Data Fig. 5 | Selected stellar evolution tracks with the time evolution of convective turnover time, $\boldsymbol{\tau}_{\boldsymbol{c}^{*}} \mathbf{a}$, Evolution in the Hertzsprung-Russell (HR) diagram from the zero age main sequence (ZAMS) to the termination age main sequence (TAMS). $\mathbf{b}$, Evolution in the HR diagram from TAMS to the red giant branch (RGB) tip. $\mathbf{c}$, Evolution of $\tau_{c}$ from ZAMS to TAMS. d, Evolution of $\tau_{c}$ from TAMS to RGB tip. All ages are normalised to TAMS or RGB tip age. 